\title{
A Study on Phonemic Analysis for the Recognition of Korean Speech
}

\author{
Jeong Young Song ${ }^{1}$, Min Wook Kil${ }^{2}$, and Il Seok Ko ${ }^{3}$ \\ ${ }^{1}$ Department of Computer Eng., Paichai University, 14 Yeonja 1Gil, DaeJeon, South Korea \\ jysong@pcu.ac.kr \\ ${ }^{2}$ Dept. of Medical Inform., Mun Kyung College, HoGyeMyun, Mun Kyung, South Korea \\ mwkil@mkc.ac.kr \\ ${ }^{3}$ Dept. of Computer and Multimedia., Dongguk University, 707 Seokjang-dong, Gyeongju, \\ South Korea \\ isko@dongguk.edu
}

\begin{abstract}
This paper is a study related to phonemic analysis of Korean speech. For speech recognition, the best method is that speech should be recognized as phonemic unit. However, to segment speech into phonemic units is not wellapplied because of various changes of phonation. Therefore, I in this study try to arrange a phonemic system of Korean speech and segment speech into phonemic units on the base of the phonemic system of Hunminjeongeum, the Korean script. I also look for the features of each phoneme by observing the changes of frequency domain, mel bandwidth and mel capstrum of phoneme and aim to form the phonemic segmentation system of Korean speech.
\end{abstract}

Keywords: phonemic analysis, pattern recognition.

\section{Introduction}

This paper is a study on phonemic segmentation of speech for recognition of Korean speech. A phoneme is the smallest unit to consist speech. Hence, speech recognition by phonemes is much more effective and efficient than recognition of syllable or word unit. It also can offer a basis of embodiment of the effective speech recognition system.

Therefore, I in this paper analyze Korean speech on phonemic system of Hunminjeongeum, the Korean script and also do a comparative analysis between features of these and frequency domain, Mel frequency band, Mel cepstrum. After that, I find out the rules that can separate Korean speech into phonemic unit by extracting phonemic features of Korean speech.

This paper analyzes the phonemic system of hunminjeongeum in chapter2 and comparative-analyzes speech into frequency domain, Mel frequency band, and Mel cepstrum according to the phonemic system of Hunminjeongeum. Chapter 4 shows the conclusions.

\section{The System of Hunminjeongeum}

Hunminjeongeum classifies speech into an initial sound, a medial vowel and a final consonant. 


\subsection{The System of Initial Sound}

Hunminjeongeum is the phonemic letters for phonetic representation. Initial sounds are classified into entire-purity, second-purity, entire-impurity and no-purity-noimpurity like Table1. They are also consisted of the sounds from molar, tongue, lip, tooth, throat, anti-tongue, and half-tooth in according to the location of speech pronounced[1]. In modern phonetics, entire-purity is classified into common-sound, second-purity into an aspirate, entire-impurity into a fortis and no-purity-no-impurity into sympathetic sound[2].

Table 1. System of Initial Sound

\begin{tabular}{|c|c|c|c|c|c|c|}
\hline $\begin{array}{l}\text { Position } \\
\text { Manner }\end{array}$ & $\begin{array}{l}\text { Back } \\
\text { teeth }\end{array}$ & Tongue & Lips & Teeth & Threat & $\begin{array}{c}\text { Semi } \\
\text { tongue }\end{array}$ \\
\hline Lax & $\neg$ 国 & $ᄃ[d]$ & $\forall[\mathrm{b}]$ & $\begin{array}{l}\text { 스[s] } \\
\text { 자i] }\end{array}$ & & \\
\hline Aspirate & $\Rightarrow[\mathrm{k}]$ & $E[t]$ & 可[p] & $ㅊ ㅏ . \mathbf{c h}]$ & $\bar{\sigma}[\mathrm{h}]$ & \\
\hline Strong & $\pi[\mathbf{k k}]$ & tr [tt] & 뻐 [pp] & $M[\mathbf{s s}] \bar{M}$ [ij] & & \\
\hline Sonorant & o[ng] & ᄂ [n] & ㅁ[m] & & & $=[1]$ \\
\hline
\end{tabular}

\subsection{The System of Medial Vowel}

A medial vowel is consisted of fundamental tone, conjunction, and wall in according to the shape of lips when they tune. There are intermediate vowels like [Ø],[r],[j],[i] which are developed in between initial sounds and medial vowels. And when $[\cdot]$ and $[\partial]$ are united with these intermediate vowels, next things happen; $[\varnothing]+[\partial] \rightarrow \cdot,[r]+$ $[\partial] \rightarrow \cdot,[j]+[\partial] \rightarrow-,[i]+[\partial] \rightarrow[1]$. that's how fundamental tones, $[\cdot,-, \mid]$, are established as Table2.

Table 2. System of Medial Vowel

\begin{tabular}{|c|c|c|}
\hline fundamental tone & conjunction[w] & wall $[\mathbf{u}]$ \\
\hline . & 上 & r \\
\hline - & $\top$ & -1 \\
\hline 1 & 표/T & 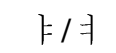 \\
\hline
\end{tabular}

If I indicate the thing that makes the shape of lips round as [w] and the thing that makes the shape of lips open as [u] when they tune, two phonemes which are 
$[\mathrm{w}]+[\cdot] \rightarrow[\perp], \quad[\mathrm{u}]+[\cdot] \rightarrow[$ F $]$ will be established. The following shows that another two phonemes which are established in $[-] ;[\mathrm{w}]+[-] \rightarrow[\top][j w \partial]$, $[\mathrm{u}]+[-] \rightarrow[\dashv][j u ̨ \partial]$. Then again, if $[$ ㄴ, $,, \top, \dashv]$ are united with intermediate vowel [i], there will be phonemes created; [ㅍ, $\pi, k, \exists][1]$.

\subsection{The System of Final Consonant}

A final consonant is that consists the rhyme of letter from receiving an initial sound and a medial vowel. There are 8 of them; ᄀ, O,ㄷ, ᄂ,ㅂ,므,,ㄹ. These are not much different from 7 of ending sounds of syllables treated as phonemics in modern phonology. Only the difference is that "ᄃ" and "入" can be treated the same in the final consonant.

\section{Phonemic Segmentation}

\subsection{Conditions}

This experiment did a sampling of speech with $16 \mathrm{kHz}, 16 \mathrm{bit}$, and Mono by using a table microphone, found out the frequency through Fourier transform. And after transforming this into Mel frequency band, Mel cepstrum was gotten.

\subsection{Phonemic Segmentation}

Speech was divided into initial sound, medial vowel and final consonant as Table3 and each of pictures shows spectrum per frequency $(\mathrm{F} / \mathrm{kHz})$, time of pronunciation(Second), Mel Frequency band(MF/melf), Mel Cepstrum(MC) of the range of $8 \mathrm{kHz}$ through Fourier transform. It finds out the sum of frequencies which are transformed into Mel frequency band and establishes the sound intervals. Again it was separated as a voiceless sound when high frequency was strong and it was separated as a voiced sound when low frequency was strong.

Table 3. Sections of Speech

\begin{tabular}{|c|l|}
\hline sections & \multicolumn{1}{c|}{ contents } \\
\hline initial sound & $\begin{array}{l}\text { entire-purity, second-purity, entire-impurity, } \\
\text { no-purity-no-impurity }\end{array}$ \\
\hline medial vowel & fundamental tone, conjunction, wall \\
\hline final consonant & $\neg, \bigcirc, \sqsubset,\llcorner$, 曰,口,(人), ᄅ \\
\hline
\end{tabular}

\subsubsection{Segmentation of Initial Sound}

An initial sound is divided up into entire-purity, second-purity, entire-impurity and no-purity-no-impurity like Figure 1 to Figure 4. 
Entire-purity of initial sound forms high frequency and has little spectrum of Mel frequency band, so it can be easily distinguished.

Second-purity ( $\exists$, E, II , ㅊ, 흐) of initial sound is an aspirated sound and forms comparatively longer time of high frequency. Especially “忈” has very strong frequency and little Mel spectrum, so it is easy to be distinguished

Entire-impurity ( 77, [ᄃ, 브, 从 즈) of initial sound is a fortis that except “从” " has a very short time to pronounce. However, it rapidly changes the spectrum of vowel coming next.

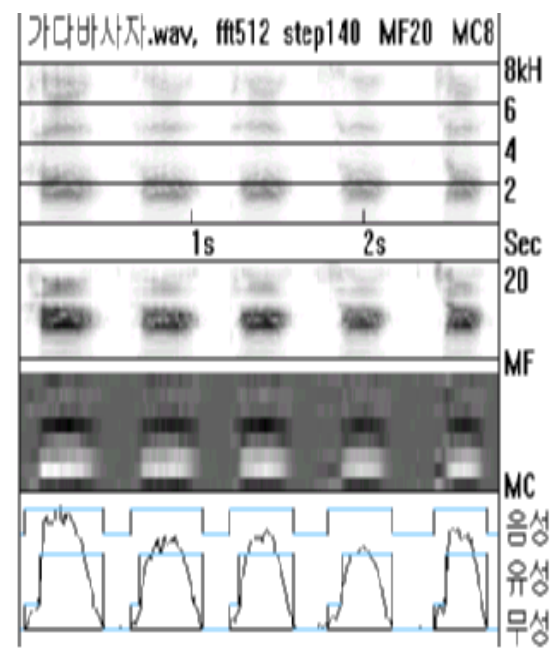

Fig. 1. Entire-Purity( ᄀ,ᄃ,曰,人,ス)

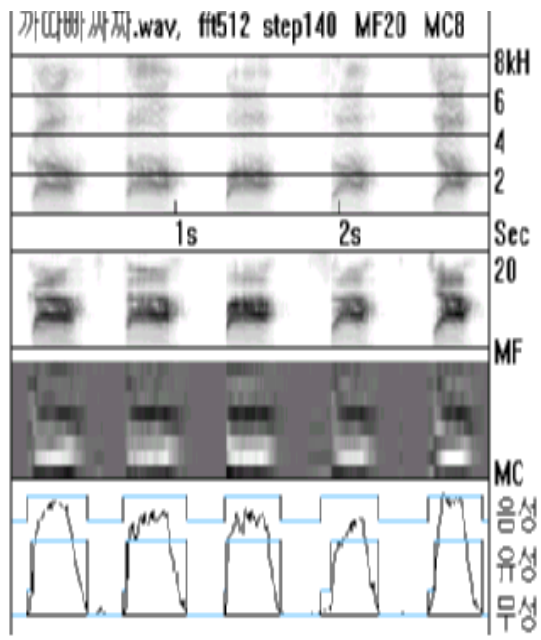

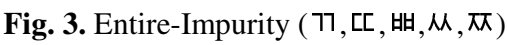

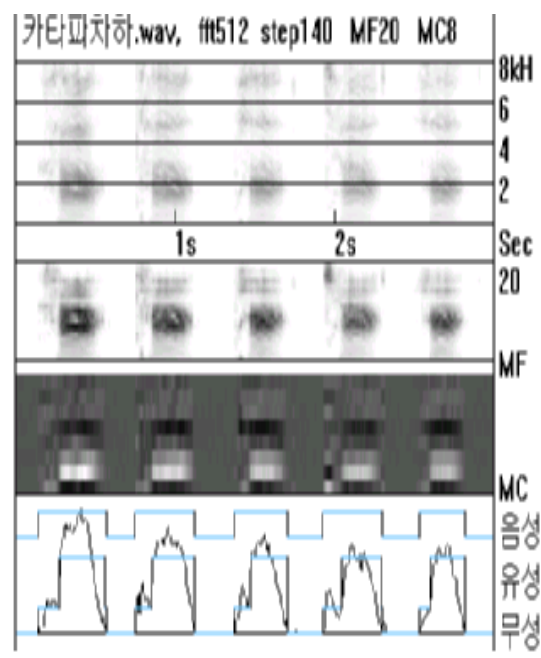

Fig. 2. Second-Purity( $($, E, $\overline{\text { ㅍ }}$, ,흐)

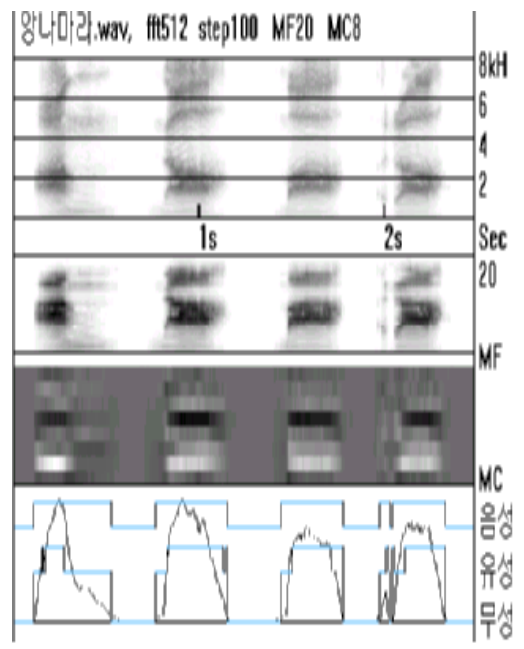

Fig. 4. No-Purity-no-impurity $(\bigcirc,\llcorner$, 口,己) 
No-purity-no-impurity( $($, ட, ロ, コ) of initial sound is a sympathetic sound. Here, "O" cannot have a sound of initial sound, but a sound of final consonant. It has an echo after vowel. You can see that “ $\llcorner$, 口, ᄅ” has a change of spectrum of vowel.

\subsubsection{Segmentation of Medial Vowel}

A medial vowel is divided into fundamental tone, conjunction in Figure 5 and wall in Figure 6. In Figure 5, “上, 丁", the conjunction of vowels, are the shapes added central formant to the spectrum of "-, I", fundamental tones, and again the spectrum gets changes when "교/T" start, then it gets stable again the forms of “上, 丁”.

In Figure 6, “F, $\dashv ”$ among wall sounds keep steady spectrum, “作” gets change from the beginning of “, $\dashv$ ” and again gets stable as forms of “, $\dashv$ ”.

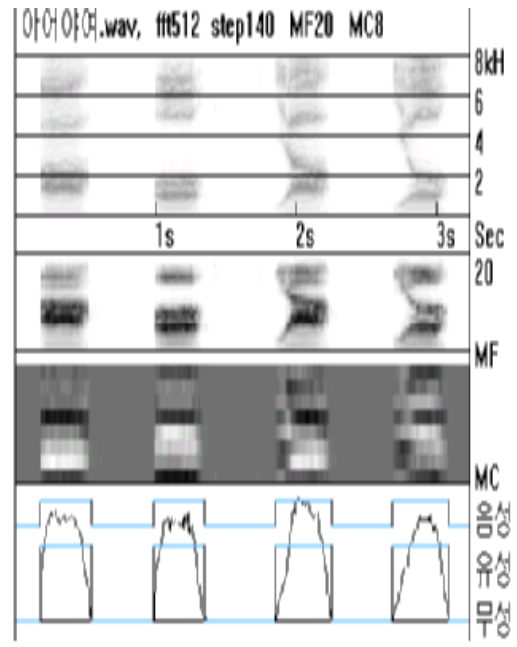

Fig. 5. Fundamental Tone and Conjunction $(一, I, \perp, T, ㅍ / 1 \pi)$

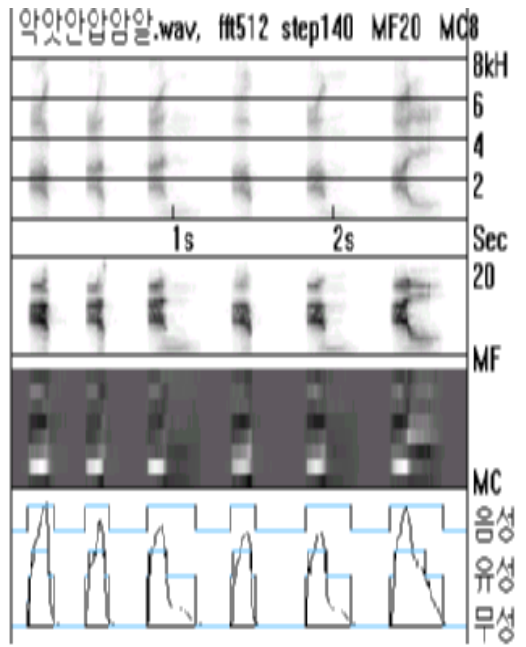

Fig. 6. Wall $(\vdash,-1, k / \neq)$

\subsubsection{Segmentation of Final Consonant}

Among 8 of final consonants, “, O, ᄃ, ᄂ, ㅂ, ㅁ, (入), ㄹ, if I treat “ᄃ” and “人” as the same sound and exclude " $O$ " shown in no-purity-no-impurity, then it will be as Figure 7.

Some of final consonant, “ᄂ, $\square$, , ”, are easy to be distinguished because they form echo, but the other, “ᄂ, $\square$, , ᄅ", hardly show the form and happen to change the spectrum of vowels as shown in Figure 7.

\subsection{Speech Experiment}

Figure 8 is the pronunciation of "안녕 하십니까" about for a second. In Figure 8, vowels, ( $\vdash, f, \vdash,||,, \mid)$, are distinguished easily like frequency and Mel 


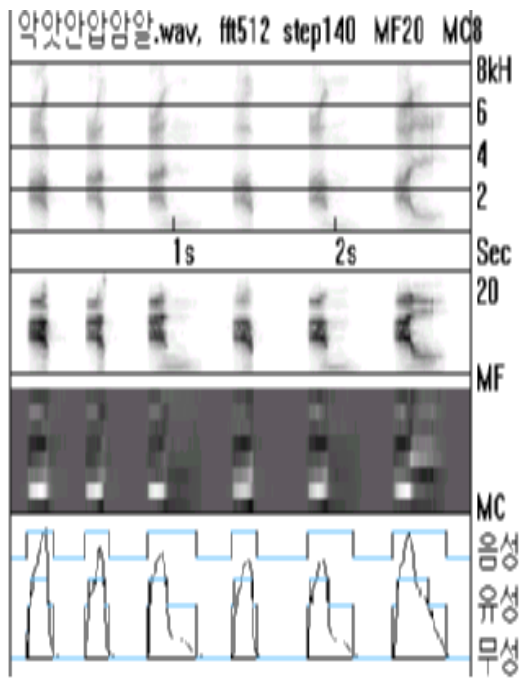

Fig. 7. Final Consonants ( ᄀ,人(ᄃ), ᄂ, 日, ロ,己)

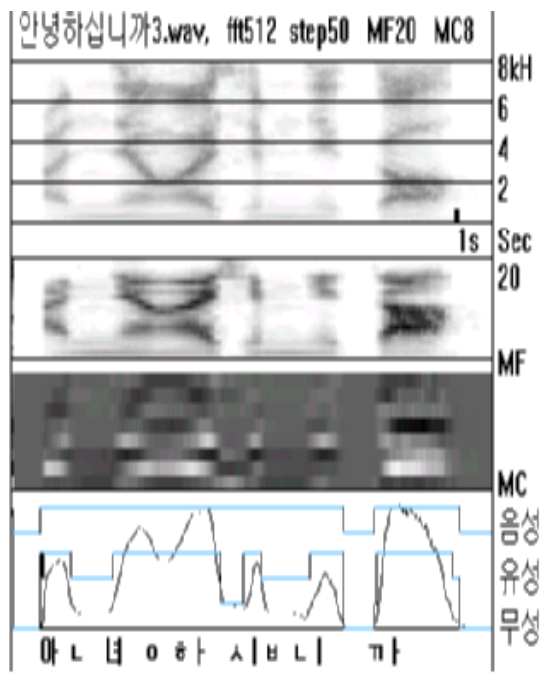

Fig. 8. An nyung ha sip ni kka

frequency band spectrum. " $\llcorner$ " should be sensed by change of spectrum and "흐" is hardly pronounced and also gets change of spectrum like in Figure 4. "人” is showing a certain high frequency as like in Figure 1 and " 77 " also gets change of spectrum as like in Figure 3.

\section{Conclusion}

I in this paper analyze the features of Korean speech in according to the phonemic system of Hunminjeongeum, the Korean script and use these features to divide speech and feature of each phoneme by using the sum of Mel cepstrum through frequency domain, Mel frequency band and DCT(Discrete Cosine Transform) transform and spectrum transformed into Mel frequency band.

Phonemes of initial sound forms high frequency or changes the spectrum and medial vowels keep the steady spectrum after changing from the fundamental tones or change the spectrum. Vowels especially can be distinguished because they keep a certain form with spectrum. Sympathetic sounds of final consonants can be distinguished because they form echo but the others can be distinguished by sensing change of spectrum.

Therefore, if there is a further study on phoneme of speech that is weakened or omitted when the sound is prolonged, it will bring more accurate phonemic segmentation.

\section{References}

1. Younghuan O, " Management of voice data," Heungryung Science Publishers, (1998).

2. Juchae Bae, "Synopsis of Hangul phonology," Shingu Culture Publishers, (1997). 
3. Lawrence Rabiner, "Fundamentals of Speech Recognition," Prentice Hall, (1993).

4. Jinsoo Han, "Management of Voice Signal," Osung Media, (2000).

5. Peter B. Denes, Elliot N Pinson, "The Speech Chain," Hanshin Culture Books, (1999).

6. Changyun Yu, "Notes on Hunminjungeum," Hyoungsul Publishers, (1998).

7. John Clark, Collin Yallop, "Phonetics \& phonology" Hanshin Culture Books, (1998).

8. Byunggon Yang, "Theory \& Practicals of Voice Analysis Using Praat," Mansu Publishers, (2003).

9. Sou-Kil Lee, Jeong-Young Song, "A Study on the Phonemic Analysis for Korean Speech," Proceeding of the Electronics, Information and Systems Conference Electronics, Information and Systems Society, I.E.E. of Japan, (2003) 